\title{
Hepatitis C Surface Antibody Measurement
}

National Cancer Institute

\section{Source}

National Cancer Institute. Hepatitis C Surface Antibody Measurement. NCI Thesaurus.

Code C74712.

The determination of the surface antibody reaction of a sample to the Hepatitis C virus. 